different types of boundary between the $L$ volumes comprising an $M$ mosaic. In some circumstances there may be a condition where $L=M$ and the particle would then be an unoriented agglomerate of crystallites.

The texture will, of course, vary profoundly from one carbon to another. In a well-crystallized (for example, natural) graphite there will be considerable 3 -dimensional near-parallel alignment of layer-groups to form mosaies closely approximating to single crystals. Larger misorientations between the mosaic sub-blocks, particularly in the $M a$ plane, would in part account for the limited ultimate crystalline development in other so-called 'graphites'.

Thus, while X-ray diffraction has the unique ability to reveal the size of the basic crystalline unit in carbons, further parameters need to be recognized when assessing the general crystalline texture of such materials and when considering their physical properties.

E. G. STeward

B. P. CoOK

Research Laboratories,

The General Electric Co., Ltd., Wembley, England.

' Houska, C. R., and Warren, B. E., J. App. Phys., 25, 1503 (1954).

2 Bacon, G. E., A.E.R.E. $M / R$ 2702, Unclassified Report, 14 (1958).

${ }^{3}$ Franklin, R. E., Acta Cryst., 4, 253 (1951).

${ }^{4}$ Bowman, 5 . C., Proc. First and Second Conf. on Carbon, 59 (University of Buffalo, 1956).

${ }^{5}$ Steward, E. G., and Cook, B. P., Z. Krist. (in the press).

- Kuroda, H., Bull. Chem. Soc., Japan, 32, 728 (1959).

'Dawson, I. M., and Follett, E. A. C., Proc. Roy. Soc., A, 253, 390 (1959).

\section{Crystal Structure of Adenosine-5'-phosphate}

ADENYLIC acid, or adenosine- $\tilde{5}^{\prime}$-phosphate, is a nucleotide of particular biochemical interest. It is not only a component of ribonucleic acid, but also forms a part of the structure of many coenzymes and, as the di- and tri-phosphate, it is frequently involved in metabolic processes. Spencer ${ }^{1}$ has reviewed the information at present available on the constituent components of the nucleotides from crystal structure analysis. In addition to the references cited by Spencer, one may also mention the brief note of Brown et $a .^{2}$ on a single projection of adenosine-3'-phosphate and the paper by Alver and Furberg ${ }^{3}$ on the structure of cytidine- $3^{\prime}$-phosphate. No nucleoside-5'-phosphate has been solved previously.

The unit cell parameters for adenylic acid are $a=$ $12 \cdot 77 \AA ., b=11.82 \AA ., c=4.88 \AA ., \quad \beta=92^{\circ} 19^{\prime}$, space group $P 2_{1}$. Unidimensionally integrated data were collected on a Wiebenga-type camera using copper radiation.

The structure was solved by three-dimensional Patterson superposition methods based on identification of the phosphorus peak in the Harker section at $v=\frac{1}{2}$. It has been refined in projection on $(010)$ and along [001] to $R$ values of $13 \cdot 7$ per cent and $13 \cdot 4$ per cent respectively. Fig. $1 A$ is an $F_{0}$ synthesis showing $e(x, y)$ at the present state of refinement, and Fig. $1 B$ is a perspective drawing of the molecule.

It may be noted here that the ribose ring is puckered in such a way that carbon $3^{\prime}$ is about $0 \cdot 5 \AA$. out of the approximate plane formed by the four remaining atoms of the ring, lending further support to Spencer's hypothesis that the preferred conformations of the ribose ring are those with either atom $2^{\prime}$ or $3^{\prime}$ out of the plane.
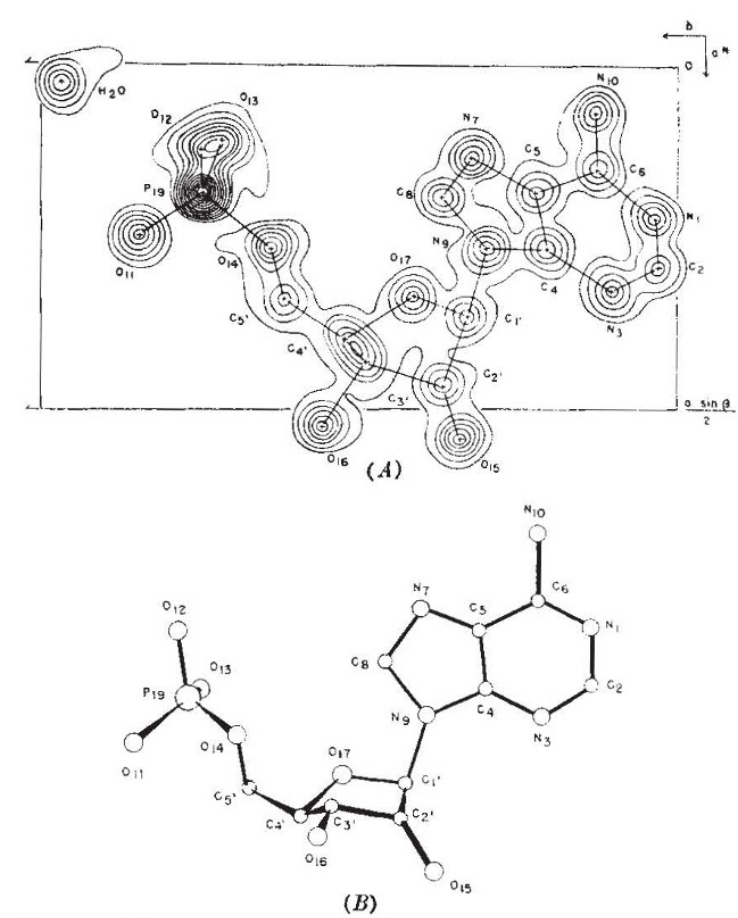

Fig. 1. (A) Fourier synthesis showing projection of electron
density along [001]; contours at intervals of $2 e \AA^{2}:^{2}(B)$ Perspective [001]; contours at intervals of $2 \mathrm{e} / \mathrm{A} .^{2} \cdot(\boldsymbol{B})$

The structure is being refined further with full three-dimensional data, and a complete account will appear elsewhere.

Department of Biochemistry,

\section{J. Kraut}

Department of Anatomy,

L. H. JENSEN

University of Washington,

Seattle 5, Washington.

${ }^{2}$ Spencer, M., Acta Cryst., 12, 59 (1959).

${ }^{2}$ Brown, D. M., Fasman, G. D., Magrath, D. I., Todd, A. R., Cochran,

W., and Woolfson, M. M., Nature, 172, 1184 (1953).

${ }^{3}$ Alver, A., and Furberg, S., Acta Chem. Scand., 13, 910 (1959).

\section{Crystal Structure of}

\section{Tetraphenylcyclobutadiene Iron Tricarbonyl}

THere has been much recent interest ${ }^{1-5}$ in the possible existence of the cyclobutadiene ring system in transition metal complexes, and a crystal structure determination $^{8}$ has verified that Criegee and Schröder's $\mathrm{C}_{8} \mathrm{H}_{12} \mathrm{NiCl}_{2}$ is indeed tetramethyl cyclobutadiene nickel dichloride. The present investigation similarly verifies the conjecture $e^{4,5,7}$ that the compound $\mathrm{Fe}(\mathrm{CO})_{3}\left(\mathrm{C}_{6} \mathrm{H}_{5} \mathrm{C}_{2} \mathrm{C}_{6} \mathrm{H}_{5}\right)_{2}$ of Hübel et al. ${ }^{4}$ is tetraphenylcyclobutadiene iron tricarbonyl.

The yellow crystals, supplied by European Research Associates, are monoclinic with $a_{0}=8.93 \AA ., b_{0}=$ $18 \cdot 73 \AA ., c_{0}=14 \cdot 10 \AA$., $\beta=92 \cdot 6^{\circ}$, with four molecules per cell, and have extinctions corresponding to space group $P 2_{1 / c}$. Diffraction intensities were obtained by the stationary crystal-stationary counter technique $e^{8}$ at rates as high as 500 a day using a General Electric $X R D-5$ spectrometer equipped with single-crystal orienter and scintillation counter. A total of 2,306 reflexions, all the reflexions with $\lambda^{-1} \sin \theta \leqslant 0.481$, were measured with a molybdenum $K \alpha$ source; of these, the strongest 1,100 have been used in the work so far. 